\title{
Structural biology: 'seeing' crystals the XFEL way
}

\author{
Vivien Marx
}
X-ray free-electron lasers (XFELs) offer opportunities beyond classic X-ray crystallography, particularly for proteins that are difficult to crystallize.

A team of scientists first painstakingly crystallizes a protein and then vaporizes the crystals. That sounds like a bad day in the lab. But in experiments with XFELs, this is exactly what researchers do to their precious crystals. This 'diffraction before destruction' is, in their view, a new way to obtain protein structures such as those of membrane proteins.

These proteins are important in signal transduction and are gatekeepers for water, nutrients or ions passing into or out of the cell. G protein-coupled receptors (GPCRs) are a large family of membrane proteins that account for around $40 \%$ of drug targets.

To explore the biological function of such proteins, scientists want structural information, which is traditionally obtained using X-ray crystallography: a few large protein crystals must be grown and are then exposed to a barrage of X-rays. Analyzing how the crystal diffracts the $\mathrm{X}$-rays helps researchers reconstruct the protein's atomic arrangement. Most atomicresolution structures of proteins have been resolved with X-ray crystallography, says

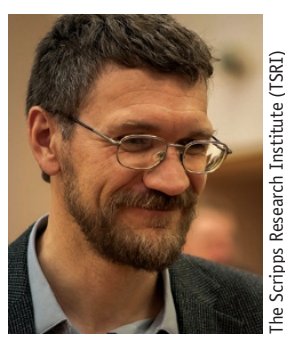

XFELs can capture a protein's transient conformations. This holds the promise of 'molecular movies,' says Vadim Cherezov.
Uwe Weierstall, a biophysicist at Arizona State University. But most membrane proteins are not readily coaxed into forming crystals.

With hydrophilic and hydrophobic domains, these proteins are tough to remove from the membrane and to keep stable. They often do not form crystals of the size, purity and regularity needed for traditional

X-ray crystallography, says Vadim Cherezov, a structural and computational biologist at The Scripps Research Institute, La Jolla.

"GPCRs are especially hard to crystallize, and often only micrometer-sized crystals can be grown," says Weierstall. They are frequently too small to be used at synchrotrons, which is where charged particles are accelerated to generate the beams for X-ray crystallography. But when

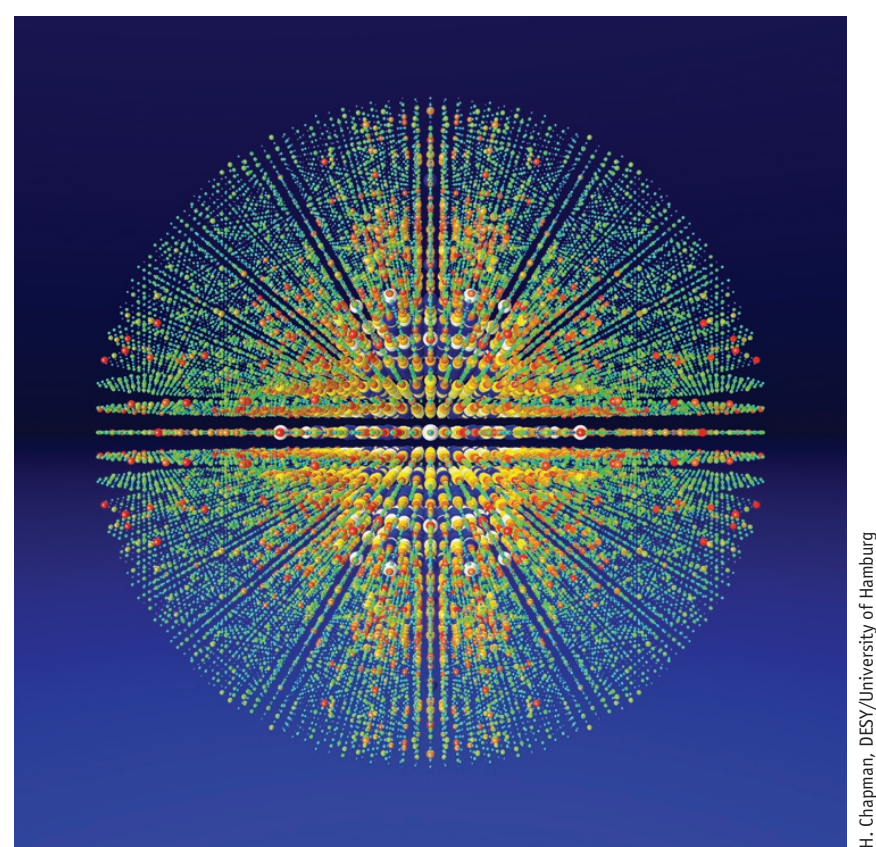

XFELs diffract before destroying a sample. Here, XFEL-based diffraction patterns from thousands of crystals are merged into a three-dimensional pattern of a suspension of crystals. structural biolo-

gists use XFELs, tiny crystals are fine.

XFELs deliver high-energy bursts of electromagnetic radiation produced in accelerators where electrons reach nearly the speed of light. Magnets force these electrons into compressed bunches, which helps them emit pulses of X-rays. An XFEL delivers the same energy in 50 femtoseconds that a synchrotron delivers in one second, says Weierstall. Fifty femtoseconds is around half the time it takes for light to traverse the width of a hair strand. Scientists can record diffraction patterns from tiny crystals shot by shot, with each shot diffracting from a crystal in a random orientation.

These bursts bring benefits, just as a flash lets a photographer take much clearer and brighter photographs and capture stop-motion and stroboscopic pictures, says Henry Chapman, who is at the Center for Free-Electron Laser Science. The center is jointly run by the Max Planck Society, the University of Hamburg and DESY, which is the German Electron Synchrotron. It is where an XFEL will go online in 2015. XFEL bursts are so fast that they let researchers capture the vibrations of atoms in a molecule. They can also visualize protein dynamics on the timescale of the XFEL pulse or longer, says Chapman.

"Cells perform the most remarkable chemistry all the time in a thermal sea of wiggling and jiggling," says biophysicist Richard Neutze of the University of 


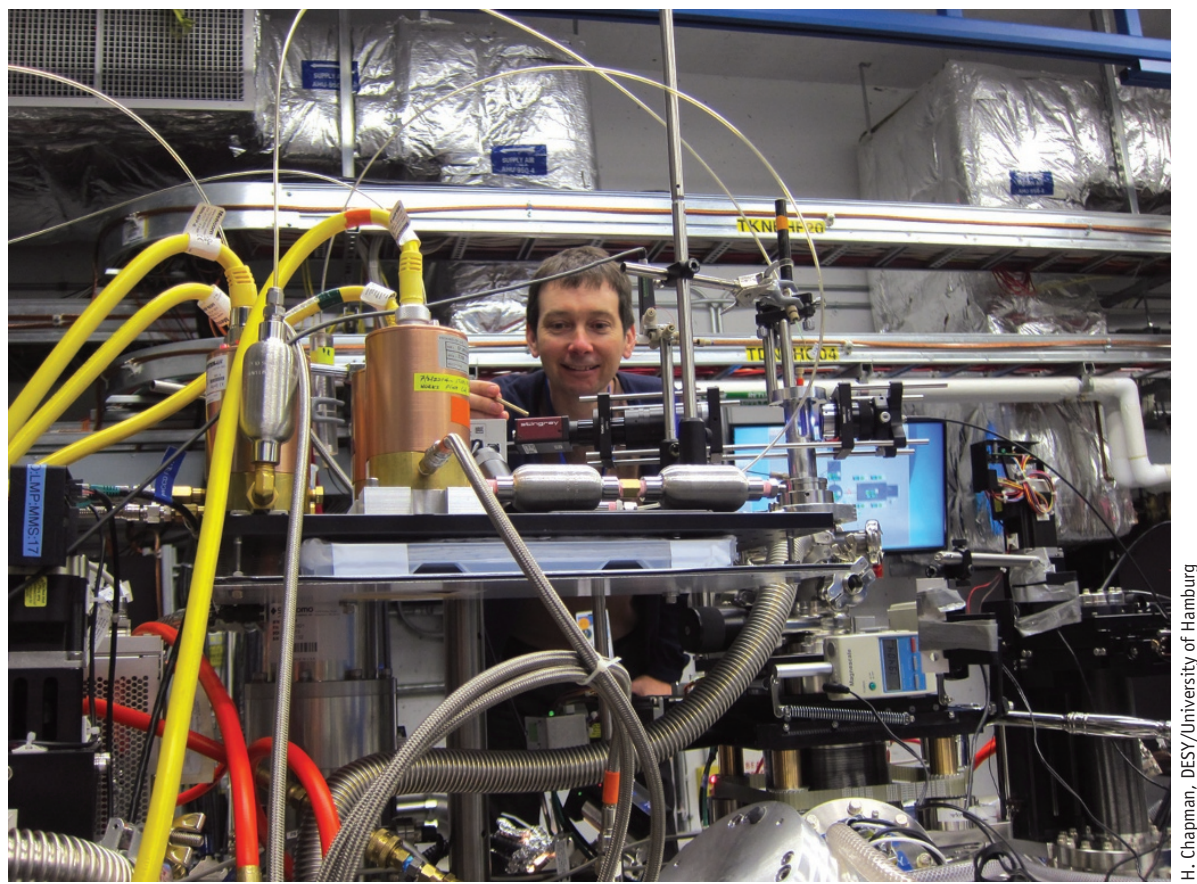

XFELs vaporize samples. But the 'glass half full' view of having a sample turn into an XFEL micro-fireball is that scientists replenish the sample for each shot, says Henry Chapman, shown here at the LCLS.

Gothenburg. Of these movements, the tiny quick ones are especially tough to capture.

Neutze and his team excited the photosynthetic reaction center of the bacterium Blastochloris viridis and then recorded X-ray scattering from a solution of protein in suspension with an XFEL. The team was able to capture conformational change in this protein that transpires in a picosecond time frame ${ }^{1}$. Previously, there had been only indirect evidence of such ultrafast changes in proteins, Neutze says. Now, researchers can explore the biological role of these motions.

In 'pump-probe' experiments, a flash of light triggers a conformational change in a protein, and the XFEL pulse collects structural data. Such transient conformational states are impossible to trap by any other available technique, says Cherezov. The approach holds the promise of recording 'molecular movies' of a protein in action, he says. "This is one of the most exciting aspects of XFELs," he says. "Eventually, with increasing XFEL power, one should be able to collect structural information from single molecules, and then crystallography will become obsolete."

\section{XFEL-ready crystals}

Scientists readying XFEL experiments explore ways to generate small crystals. "One of the most amazing ways to get small crystals is by letting them grow in the very cells where the protein is overexpressed," says Chapman. He and his colleagues used such in vivo-grown crystals to solve the structure of the protease cathepsin B from the parasite that causes African sleeping sickness, Trypanosoma brucei. In this work, they performed baculovirus infection of the Sf9 insect cell line. By coincidence they discovered that the cells produce tiny crystals of their own accord. A single cell can produce only so much protein. The size is just right for XFELbased experiments, Chapman says.

In this insect cellular environment, the enzyme likely has the post-translational modifications that it has in the parasite, he says. There is a propeptide that is initially formed as part of the protein, but it is then cleaved apart. It is this broken-off bit that inhibits the action of the protein. The team can therefore see conformational changes of the enzyme's "inhibited state," which is useful in the search for an inhibitor, a potential drug.

Structures guide research at Heptares Therapeutics. As Fiona Marshall, the company's chief scientific officer, explains, it helps to have solved a structure along with one in which a molecule such as a potential drug is attached to the receptor because small structural differences can affect how molecules might bind. She and her colleagues used traditional X-ray crystallography to solve the structure of a class C GPCR 


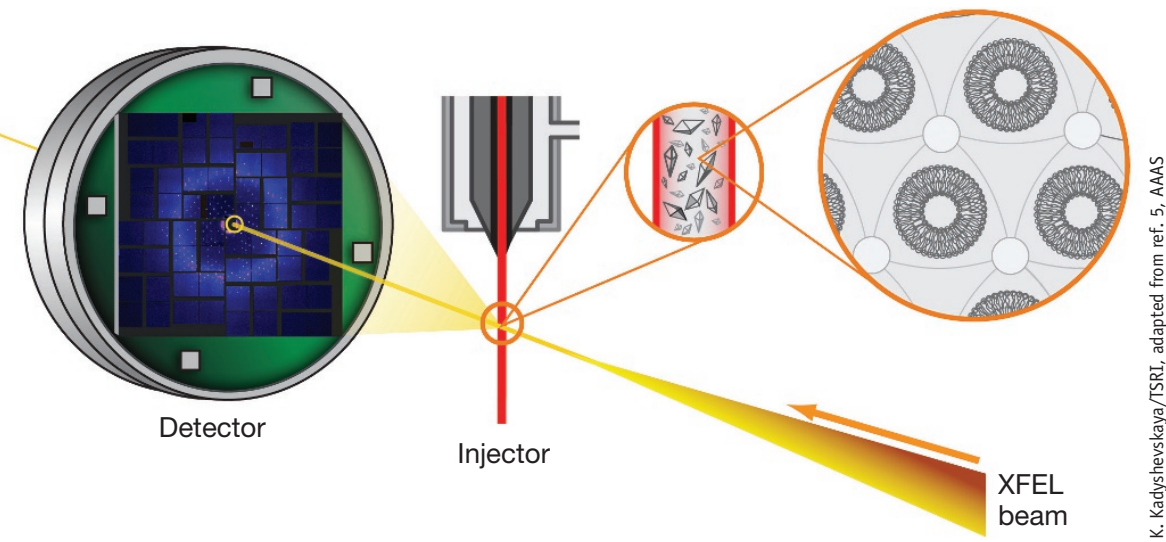

In 'diffraction before destruction' experiments, microcrystals are injected to an XFEL's bright X-ray pulses that last femtoseconds. An injector that delivers crystals in a gel-like matrix called lipidic cubic phase helps to tune the rate of crystal flow to the pulse.

domain attached to mavoglurant, a Novartis drug candidate to treat fragile $\mathrm{X}$ syndrome, which can cause mental disability or autistic behaviors ${ }^{2}$.

This structure is one of eight receptors in a family, and having it solved will help with modeling the other members and potentially accelerate drug discovery, says Marshall. Although her team did not use XFELs, she says they are "an exciting development" in $\mathrm{X}$-ray structure determination and notes their use to solve GPCR structures. "A particular advantage is that data can be collected from very small crystals," she says.

In her view, structure-based drug design needs to draw on multiple structures of molecules bound to receptors. Given that XFELs can be used to obtain such ligand costructures, the technology promises to help medicinal chemists tailor the chemical structure and properties of a potential drug, she says.

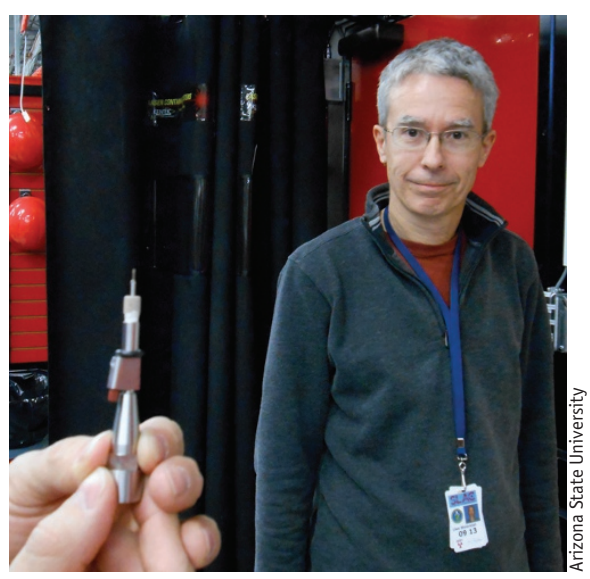

Inventor plus invention. Uwe Weierstall and colleagues have developed injectors to replenish crystals in the XFEL beam.

\section{Diffract before destroy}

These days it has become easier to pique the interest of structural biologists about XFELs, says Chapman. The skepticism about the expense and the value of XFELs for biol$\mathrm{ogy}^{3}$ has died down. XFEL experiments and instrumentation are still being optimized, but scientists see plenty of advantages in using them. For example, researchers can use a stream of tiny crystals that would not work with a synchrotron. "It certainly increases the resolution that you can obtain from poorly diffracting or small crystals," says Chapman.

Another advantage arises when doing experiments that involve activating a protein moments before the XFEL pulse arrives. The protein does not need to be returned to its ground state for a next measurement as is needed at a synchrotron, says Chapman.

True, the pulse destroys the sample, which does not sound like an advantage. But even though the pulse completely vaporizes the protein crystal, scientists obtain a strong diffraction pattern. The duration of the pulse is so short that atoms cannot move in that time, says Chapman. "In fact, the crystal is already destroyed before the light has traveled the distance to the diffraction camera."

In traditional X-ray crystallography, samples must be frozen, but this is not so for XFELs. That is an advantage because it keeps the diffracting protein crystal in a more natural environment and permits diffraction of light-induced or chemically induced conformational changes to the protein, says Weierstall.

"It is still early days in this field, and the jury is still out on whether XFEL measurements are completely 'free' of radiation damage," he says. Scientists have 


\section{BOX 1 GETTING THE MOST FROM A BEAM}

An increasing number of scientists want to try XFELs, of which there are currently only two: the SLAC LCLS in the USA and SPring-8 Angstrom Compact Free Electron Laser (SACLA) in Japan. "Getting beam time at an XFEL is becoming more and more competitive," says Weierstall.

Twice annually, the LCLS issues calls for beam-time proposals to the community, and they are peer-reviewed. Each one might involve five 12-hour shifts. The approval rate for proposals is between $18 \%$ and $22 \%$ and depends on the cycle, the length of the runs and the demand, says LCLS staff scientist Sébastien Boutet.

\section{Protein crystal screening}

In March of 2013, the LCLS began offering scientists short runs of a few hours for backto-back experiments over several days. Deemed a success, the protein crystal screening program with its six-hour beam-time blocks is now an LCLS fixture.

The program lets researchers test samples or preparation methods before committing a regular slot. "This can be useful for time-resolved experiments where it is very valuable to first make sure the crystals are good before spending effort on the time-resolved measurements," says Boutet.

A six-hour time slot at the beamline might also yield enough data to solve a structure. Or it can help scientists assess their extremely small crystals. Just having a few good diffraction patterns can deliver important data for researchers preparing a full proposal, says Boutet.

\section{Beam splitting}

The possibility to multiplex beams for different experiments was built into the LCLS design, says Boutet, and it draws on synchrotron experience. But assuring high-quality optics is a challenge. "We do this with thin crystals that take a small part of the X-ray spectrum to one experiment and let the rest pass through to a second experiment," he says.

Researchers avidly explore multiplexing, with higher demand for LCLS beam time coming from structural biologists than from other disciplines. Not every experiment can be multiplexed, but, Boutet says, beam splitting has increased the number of experiments by $15-25 \%$.

\section{More XFELs}

It will help the structural biology community when more XFELs come online, such as the ones planned in Germany, Switzerland and South Korea, says Weierstall.

For now, the work that goes into an experiment and the team it requires are both large: biologists who make the micro- and nanocrystals; an injector team responsible for sample delivery into the beam; a data analysis team who reacts as the data come in at 120 patterns per second and who advises the sample team on whether, for example, the hit rate is high enough or whether the sample should be changed; and the beamline scientists who make sure that the $X$-ray beam hits the sample and that everything runs smoothly, says Weierstall. As the technology matures, team size may be able to shrink.

compared high-dose XFEL measurements on micrometer-sized protein crystals to very low-dose synchrotron measurements on large crystals of the same protein. They find that the high X-ray dose of the XFEL applied in a few femtoseconds is not damaging the crystals more than the low dose in synchrotron measurements that is applied in seconds.

What traditional approaches and XFELbased ones share is the need to stabilize membrane proteins once they have been removed from the membrane. One way is to reconstitute them in a synthetic gel-like matrix called lipidic cubic phase (LCP), designed to make them "feel more at home," says Cherezov. LCP was developed nearly two decades ago $^{4}$, but crystallizing proteins directly from this matrix has caught on only in recent years, says Cherezov. Over the last few years, around a quarter of all new membrane structures have been obtained using LCP crystallization, he says.

In traditional crystallography, diffractions from one large crystal are used; the crystal is rotated to record different diffraction patterns. Given that XFELs destroy the crystal after each pulse, scientists need many tiny crystals in random orientation to obtain a complete data set. 
They can feed these many crystals into a beamline in a process called serial femtosecond crystallography (SFX). Tens to hundreds of thousands of crystals are needed so that the XFEL beam can hit them. That means tens to hundreds of milligrams of crystallized protein when streamed with a liquid injector, which is a difficult feat with membrane proteins, says Cherezov. $\mathrm{He}$ and his colleagues obtained a GPCR structure-the human serotonin 5-HT2B receptor-with a different kind of data collection approach, such that crystals were injected into the XFEL within the gel-like $\mathrm{LCP}^{5}$. They slowed down the crystal flow and reduced the amount of protein needed for such experiments. At the SLAC Linac Coherent Light Source (LCLS), they captured over 4 million diffraction patterns in ten hours using around 0.3 milligrams of protein, which had been crystallized in LCP.

The need for many crystals is connected to a host of factors, says Cherezov, such as unknown crystal size, the orientation of each crystal and the effect of any crystal irregularities. Both X-ray energy and beam intensity fluctuate from shot to shot such that data must be assembled with Monte Carlo algorithms. The fluctuations cancel out as the number of crystals rises, Cherezov says.

Each shot vaporizes a crystal, but, as Chapman explains, the 'glass half full' view of vaporizing a sample in an XFEL microfireball is that scientists replenish the sample for each next shot. "This means we always collect data from a fresh sample," he says. Building up a full three-dimensional structure of a protein takes at least 10,000 diffraction patterns.

XFELs produce faster pulses than a synchrotron. The LCLS delivers 120 pulses per second, which means 120 potential diffraction patterns from 120 crystals, says Chapman. "This adds up quickly, giving almost half a million diffraction patterns per hour."

Current detectors can cope with this much data, but the new European XFEL will send 30,000 pulses a second such that researchers can make measurements in seconds or minutes that took hours or days at the LCLS, says Chapman. "Of course, it's an even bigger challenge for the detectors to keep up," he says.

\section{Injecting into the beam}

There are a number of ways to inject crystals into the XFEL beam. The gas dynamic virtual nozzle (GDVN) was the first injector used for SFX and still remains the most popular, says Cherezov. It sends crystals suspended in solution into the beam in a stream a few micrometers in diameter.

But the stream's flow rate exceeds the XFEL pulse repetition rate. For every crystal that is hit and diffracted, tens of thousands of crystals pass between XFEL pulses and are wasted, says Cherezov, in addition to the challenge of needing much starting material. LCP helps solve this problem by acting as a "carrier matrix." Its viscosity and gel-like consistency allow the crystal flow rate to be fine tuned to the XFEL pulse repetition rate so that crystals can be used more efficiently.

LCP is not compatible with all types of crystals and crystallization conditions, which is why other injectors are being developed, such as electrospinning and ultrasound-based approaches, each with its own limitations, Cherezov says.

Weierstall and his team have developed different types of injectors to replenish crystals at the rate of XFEL pulses. Although the GDVN, also from his lab, is still widely used, he acknowledges the mismatch between flow rate and XFEL pulse rate and its need for large amounts of material. This challenge led him and his team to develop the LCP injector, which achieves an injection speed that matches the X-ray pulse repetition rate, says Weierstall ${ }^{6}$.

The injector consists of a hydraulic stage, a sample vessel and a nozzle, and its use delivers all the crystals contained in the LCP to the XFEL. With this injector, only around 0.5 milligrams of purified protein are needed for one structure determination, he says. Arizona State University filed a patent on this device, which has not yet been licensed, he says.

\section{Wish list for the future}

XFELs stand to change structural biology, and other technologies will, too, such as cryo-electron microscopy, says Tamir Gonen, who is at the Howard Hughes Medical Institute's Janelia Farm Research Campus. The infrastructure for cryo-EM is cheaper and more accessible than that for XFELs, he says, and the crystals can be even smaller. Structures can be determined from a single crystal and data processed with standard X-ray crystallography software.

He has developed-and recently improved-MicroED ${ }^{7,8}$, an approach in which nearly 100 diffraction patterns are collected from the same crystal. The crystal is placed in a transmission electron cryo-microscope that is used with a reduced electron dose.

In Cherezov's view, XFELs and SFX are still maturing. On his wish list for XFELs are higher beam intensities, faster pulse repetition rates, a larger energy range, and less energy jitter and intensity fluctuations. Faster detectors with a higher dynamic range will increase the quality of captured data. Cherezov also sees a need for better data processing algorithms. New methods of sample delivery beyond injectors are emerging, too, he says, such as approaches in which samples are deposited on a solid support.

Weierstall's wish list also includes continued development of detectors and sample delivery methods, improved software and additional techniques to grow nanocrystals.

The new European XFEL will save on nanocrystal slurry that is currently wasted, reducing the amount of material needed from a single milliliter to a few tens of microliters of slurry, which can be diffracted more quickly, says Chapman. Other methods development is aimed at devising dynamics experiments and attempting to 'outrun' radiation damage, especially in proteins with metal centers, says Chapman.

The field is developing quickly with scarce facilities that, says Chapman, cause a bottleneck and too little opportunity to optimize experiments and methods. Having more facilities will help, as will using the beams in more efficient ways (Box 1).

"The experiments conducted at XFELs currently are very exciting, the environment is very dynamic and the developments occur at a very high pace; therefore, it is quite possible that some of our wishes and dreams may come true much faster than anticipated," says Cherezov.

1. Arnlund, D. et al. Nat. Methods 11, 923-926 (2014).

2. Doré, A.S. et al. Nature 511, 557-562 (2014).

3. Henderson, R. Nature 415, 833 (2002).

4. Landau, E.M. \& Rosenbusch, J.P. Proc. Natl. Acad. Sci. USA 93, 14532-14535 (1996).

5. Liu, W. et al. Science 342, 1521-1524 (2013).

6. Weierstall, U. et al. Nat. Commun. 5, 3309 (2014).

7. Shi, D., Nannenga, B.L., Iadanza, M.G. \& Gonenet, T. elife 2, e01345 (2013).

8. Nannenga, B.L., Shi, D., Leslie, A.G.W. \& Gonen, T. Nat. Methods 11, 927-930 (2014).

Vivien Marx is technology editor for Nature and Nature Methods

(v.marx@us.nature.com). 